\title{
Nonparametric modeling of the variability of vehicle vibroacoustic behavior
}

\author{
Jean-François DURAND, Laurent GAGLIARDINI \\ PSA- Peugeot-Citroën- FRANCE \\ Christian SOIZE \\ University of Marne-La-Vallée- FRANCE
}

Copyright $@ 2005$ SAE International

\begin{abstract}
In order to improve the robustness of vibroacoustic numerical predictions, one introduces a model of random uncertainties. The random uncertainty modelling relies on a nonparametric approach providing random system realizations with a maximum entropy. This approach only requires a few uncertainty parameters but takes into account data errors as well as model errors. It appears to be well adapted to study the variability of structural-acoustic systems; the implementation of the method for this class of problem is presented here for the first time. Practically, the paper deals with a classical low frequency vibroacoustic modelling such as used for booming noise predictions. The application of the nonparametric approach to vehicle uncertainties modelling shows the sensitivity of the vibroacoustic frequency responses to structural and cavity uncertainties as well as coupling interface uncertainties. Flexible parts appear to be more sensitive to random uncertainties than stiff parts. The sensitivity of the structural modes to structural random uncertainties is also shown in a stochastic MAC table.
\end{abstract}

\section{INTRODUCTION}

In the automotive industry, numerical simulation is intensively used to predict the dynamical behavior of vehicles. The problem of the predictability of the models is especially difficult, due to the over-sensitiveness of dynamical responses. This variability is induced by structural complexity as much as by uncertainties resulting from the industrial process or from vehicles diversity. This over-sensitiveness may be seen numerically when applying design changes, but also experimentally when monitoring vehicles dispersions $[1,2,3,4]$. In order to improve the robustness of the predictions, one has to account for these numerous uncertainties in numerical computations. Classically, parametric probabilistic approaches are used, such as stochastic finite elements methods which constitute a very efficient tool in computational mechanics [5].
Uncertainties can be classified into two classes:

(i) Data uncertainties concern the parameters of the mathematical-mechanical model such as geometrical parameters, boundary conditions, material behaviour etc. Data uncertainties can be described by a parametric probabilistic approach. Nevertheless, in the case of vehicles, such an approach requires a considerable amount of statistical data which makes it inappropriate to an industrial design process.

(ii) Model uncertainties are introduced during the construction of the mathematical-mechanical model: the constructed model cannot exactly represent a structure as complex as a car body due to the introduction of approximations and simplifications which make the modelling practicable. This class of uncertainties is not relevant to the parametric approach because, by definition, the model uncertainties cannot be taken into account by the parameters of the mathematicalmechanical model under consideration. It has been seen many times that small design changes -that may be considered as model uncertainties- lead to much higher differences in dynamic responses than any observed probabilistic parametric responses. This high sensitivity leads to the conclusion that a large part of the lack of predictability is due to model uncertainties.

Recently, a new approach, called the nonparametric approach, has been introduced to model random uncertainties in linear and non-linear elastodynamic problems, using the maximum entropy principle $[19,20,21]$. This principle ensures that any possible change, within a given spread, will be covered by the random uncertainty model. Consequently, random data as well as random modelling changes are handled by the method. This paper is the first application of the nonparametric method to the structural-acoustic problem.

Considering the large number of small variations by which a vehicle may be affected -during the development process and later during the manufacturing 
process-, the modelling of uncertainties by a stochastic process seems convenient. The proposed approach allows the direct construction of a probabilistic model of matrices representing the mechanical behaviour. Only one parameter is required per matrix. It defines the range of random changes to be modelled. Of course the value of the uncertainty parameters is an important issue, but it will not be discussed yet.

In this early paper, we will only present the numerical implementation of the method in the case of a vibroacoustic problem and show the sensitivity of a trimmed body model response to such random uncertainties.

Structural uncertainties, acoustic cavity uncertainties and coupling interface uncertainties are introduced. Vibroacoustic random responses to a known powertrain excitation (booming noise) are computed at structural points and at the driver's ears. The associated confidence regions are numerically constructed, following a Monte-Carlo process. Structural modes sensitivity to structural random uncertainties is also plotted as a stochastic MAC table.

But first, we will recall the statement of the structural acoustic problem, that is commonly solved in order to simulate the vibro-acoustic response of car bodies. Later on, the construction of the stochastic structural acoustic problem, introducing the non-parametric modelling of uncertainties, will be explained.

\section{STATEMENT OF THE STRUCTURAL-ACOUSTIC PROBLEM IN THE FREQUENCY DOMAIN}

Let us consider linear vibrations of a damped structure $\Omega_{S}$ subjected to external loads, coupled with its internal cavity $\Omega_{F}$. We are interested in predicting the frequency responses of the structural-acoustic system in the frequency band of analysis $\left[\omega_{\min }, \omega_{\max }\right]$.

\section{GEOMETRY AND MECHANICAL ASSUMPTIONS}

The physical space $R^{3}$ is referred to a cartesian reference system and we denote the generic point of $R^{3}$ as $\mathbf{x}=\left(x_{1}, x_{2}, x_{3}\right)$. We study the linear vibration of a structural-acoustic system around a static equilibrium state taken as natural state at rest.

\section{$\underline{\text { Structural part }}$}

The structural part occupies a three-dimensional bounded domain $\Omega_{S}$ of $R^{3}$ with a sufficiently smooth boundary $\partial \Omega_{S}=\Gamma$. The outward unit normal to $\partial \Omega_{S}$ is denoted as $n_{S}=\left(n_{S_{1}}, n_{S_{2}}, n_{S_{3}}\right)$. The displacement field in $\Omega_{S}$ is denoted as $\mathbf{u}(\mathbf{x}, \omega)=\left(\mathrm{u}_{1}(\mathbf{x}, \omega), \mathrm{u}_{2}(\mathbf{x}, \omega), \mathrm{u}_{3}(\mathbf{x}, \omega)\right)$. The structure is assumed to be free, i.e. not attached on any part of its boundary $\partial \Omega_{S}$.

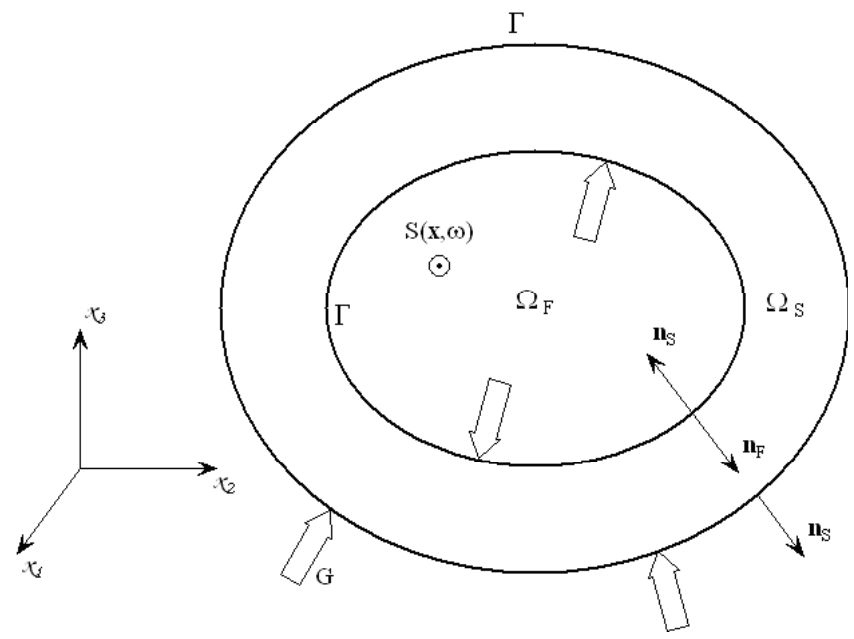

Figure1 Configuration of the structural-acoustic system

Internal acoustic cavity

The internal acoustic cavity $\Omega_{F}$ is the bounded domain filled with an internal acoustic dissipative fluid. The boundary $\partial \Omega_{F}$ of $\Omega_{F}$ is again $\Gamma$. The outward unit normal to $\partial \Omega_{F}$ is denoted as $\mathrm{n}_{\mathrm{F}}=\left(\mathrm{n}_{\mathrm{F} 1}, \mathrm{n}_{\mathrm{F} 2}, \mathrm{n}_{\mathrm{F} 3}\right)$ and we have $\mathbf{n}_{\mathrm{S}}=-\mathbf{n}_{\mathrm{F}}$ on $\partial \Omega_{F}$. We denote the pressure field in $\Omega_{F}$ as $\mathrm{p}(\mathbf{x}, \omega)$.

\section{BOUNDARY VALUE PROBLEM OF THE STRUCTURAL-ACOUSTIC SYSTEM -}

The equation of the structural part occupying domain $\Omega_{S}$ is written $[6,7,8]$

$$
-\omega^{2} \rho_{S} u_{i}-\sigma_{i j, j}=g_{i}^{v o l} \text { in } \Omega_{S}
$$

in which $\rho_{S}$ is the mass density, $\sigma_{i j}$ is the stress tensor, $\mathbf{u}(\mathbf{x}, \omega)=\left(u_{1}(\mathbf{x}, \omega), u_{2}(\mathbf{x}, \omega), u_{3}(\mathbf{x}, \omega)\right)$ is the displacement field of the structure and $\mathbf{g}^{\text {vol }}(\mathbf{x}, \omega)=\left(g_{1}{ }^{\text {vol }}(\mathbf{x}, \omega), g_{2}{ }^{\text {vol }}(\mathbf{x}, \omega)\right.$, $\left.\mathrm{g}_{3}{ }^{\mathrm{vol}}(\mathbf{x}, \omega)\right)$ is the body force field.

The boundary conditions can be written as

$$
\sigma_{i j} u n_{S, j}=g_{i}^{\text {surf }}-p n_{S, i} \text { on } \Gamma
$$

where $g^{\text {surf }}$ is the surface force field and $p$ is the pressure at the fluid coupling interface. The material is assumed to be linear viscoelactic without memory which allows the constitutive equations to be defined.

Concerning the internal acoustic fluid, a formulation in terms of pressure is used. The equation governing the dynamics of the fluid is written as $[9,10,11,12]$ : 
$\frac{\omega^{2}}{\rho_{F} c_{F}{ }^{2}} p+i \omega \frac{\tau}{\rho_{F}} \nabla^{2} p+\frac{1}{\rho_{F}} \nabla^{2} p=\frac{\tau}{\rho_{F}} c_{F}^{2} \nabla^{2} S-i \frac{\omega}{\rho_{F}} S$ in $\Omega_{S}$

and,

$\frac{1}{\rho_{F}}(1+i \omega \tau) \frac{\partial p}{\partial \mathbf{n}_{\mathbf{F}}}=\omega^{2} \mathbf{u} . \mathbf{n}_{\mathbf{F}}+\tau \frac{c_{F}^{2}}{\rho_{F}} \frac{\partial S}{\partial \mathbf{n}_{\mathbf{F}}}$ on $\Gamma$,

where $\rho_{F}$ is the mass density of the fluid, $c_{F}$ is the speed of sound, $\tau$ represents the coefficient due to the viscosity of the fluid, and $S(x, \omega)$ is the source term.

\section{FINITE ELEMENT DISCRETIZATION}

The finite element method [13] is used to solve numerically the above boundary value problem. We consider a finite-element mesh of the structure $\Omega_{S}$ and of the internal fluid $\Omega_{F}$. Let $\underline{U}^{S}=\left\{\underline{U}_{1}{ }^{S}, \ldots, \underline{U}_{n s}{ }^{S}\right\}$ be the complex vector of the ns degrees of freedom (DOF) of the structure corresponding to the finite element discretization of the displacement field $u$. Let $\underline{P}^{F}=\left\{\underline{P}_{1}^{F}, \ldots, \underline{P}_{n f}{ }^{F}\right\}$ be the complex vector of the nf DOF of the fluid corresponding to the finite element discretization of the pressure field $p$. Therefore, the finite element discretization of the boundary value problem in terms of $\mathbf{u}$ and $p[9,12,14]$, defined by Eqs.(1) to (4) yields the following matrix equation

$$
\left[\begin{array}{cc}
\underline{A}_{S}(\omega) & \underline{C} \\
\omega^{2} \underline{C}^{T} & \underline{A}_{F}(\omega)
\end{array}\right] \underline{\underline{P}}^{F}(\omega)=\left[\begin{array}{l}
\underline{F}^{S}(\omega) \\
\underline{S}^{F}(\omega)
\end{array}\right]
$$

where $\underline{A}_{S}(\omega)$ is the dynamical stiffness matrix of the structure such that $\underline{A}_{S}(\omega)=-\omega^{2} \underline{M}^{S}+i \omega \underline{D}^{S}+\underline{K}^{S}$ and where $\underline{M}^{S}, \underline{D}^{S}, \underline{K}^{S}$ are respectively the mass, damping and stiffness matrices of the structure in vacuo.

In Eq.(5), $\underline{A}_{F}(\omega)$ is the dynamical stiffness matrix of the acoustic fluid such that $\underline{A}_{F}(\omega)=-\omega^{2} \underline{M}^{F}+i \omega \underline{D}^{F}+\underline{K}^{F}$ in which $\underline{M}^{F}, \underline{D}^{F}, \underline{K}^{F}$ are the mass, damping and stiffness matrices of the cavity with fixed coupling interface. $\underline{C}$ is the vibroacoustic coupling matrix.

Introducing the change of variable $\underline{P}^{F}(\omega)=i \omega \underline{\widetilde{P}}^{F}(\omega)$, Eq.(5) can be rewritten as the following symmetric matrix equation,



Eq.(6) is a highly sparse linear system which size is the total number of DOFs considered.

\section{REDUCED MATRIX MODEL OF THE STRUCTURAL- ACOUSTIC SYSTEM}

The structural modes in vacuo and the acoustic modes of the cavity with fixed coupling interface are calculated by solving the two generalized eigenvalue problems,

$$
\begin{aligned}
& \underline{K}^{S} \underline{\psi}=\underline{\lambda}^{S} \underline{M}^{S} \underline{\psi} \\
& \underline{K}^{F} \underline{\varphi}=\underline{\lambda}^{F} \underline{M}^{F} \underline{\varphi}
\end{aligned}
$$

The eigenvectors verify the usual orthogonal properties $[15,16,17,18]$. The structure in vacuo has six rigid body modes corresponding to a zero eigenvalue and $n-6$ elastic modes. Since we are only interested in the elastic deformation of the structure, the structural displacement is written as

$$
\underline{\mathbf{U}}^{S}(\omega)=[\underline{\Psi}] \underline{q}^{S}(\omega)
$$

in which $[\Psi]$ is a matrix whose columns are constituted of the $n$ elastic structural modes associated with the $n$ first positive eigenvalues, and $q^{S}$ is the vector of the structural generalized coordinates.

The internal acoustic cavity has one constant pressure mode and m-1 acoustic modes. The internal acoustic pressure is written as

$$
\underline{\mathbf{P}}^{F}(\omega)=i \omega[\Phi] \underline{\tilde{q}}^{F}(\omega)
$$

in which $[\Phi]$ is the matrix whose columns are constituted (1) of the constant pressure mode associated with zero eigenvalue and (2) of the acoustic modes associated with the positive eigenvalues (the $m-1$ first acoustical eigenfrequencies), and $\widetilde{q}^{F}$ is the vector of acoustic generalized coordinates.

It should be noted that the constant pressure mode is kept in order to model the quasi-static variation of the internal fluid pressure induced by the deformation of the coupling interface [9]. Using Eqs.(9) and (10), the projection of Eq.(6) yields the mean reduced matrix model of the structural-acoustic system,

$$
\left[\begin{array}{l}
\underline{\mathcal{A}}^{S}(\omega) i \omega \underline{C} \\
\left.i \omega \underline{C}^{T}-\underline{\mathcal{A}}^{F}(\omega)\right] \underline{q}^{S}
\end{array}=\left[\begin{array}{c}
\underline{F}^{S}(\omega) \\
-\frac{1}{i \omega} \underline{S}^{F}(\omega)
\end{array}\right]\right.
$$

The generalized dynamical stiffness matrix of the structure is then written as

$$
\underline{\mathcal{A}}^{S}(\omega)=-\omega^{2} \underline{\mathcal{M}}^{S}+i \omega \underline{\mathcal{D}}^{S}+\underline{\mathcal{K}}^{S}
$$

The generalized dynamical stiffness matrix of the cavity is then written as

$$
\underline{\mathcal{A}}^{F}(\omega)=-\omega^{2} \underline{\mathcal{M}}^{F}+i \omega \underline{\mathcal{D}}^{F}+\underline{\mathcal{K}}^{F}
$$


Eq.(11) is a reduced size linear system; typically, a few thousands of modes are considered. Moreover, generalized dynamical stiffness may be diagonal, when an appropriate damping model is chosen. It will be shown later why these properties are of great importance regarding the implementation of the proposed method.

\section{CONSTRUCTION OF THE STOCHASTIC STRUCTURAL-ACOUSTIC PROBLEM WITH THE NONPARAMETRIC APPROACH OF UNCERTAINTIES}

The uncertainties of predictions are induced by data errors and by modelling errors. Due to the presence of model errors, the usual parametric probabilistic approach which is perfectly adapted to model data errors, cannot be used. Consequently, we proposed a non-parametric probabilistic model of random uncertainties allowing data errors and model errors to be taken into account. Such a non-parametric probabilistic model has been proposed in Refs. [19, 20, 21] by one of the authors. Until now, it has only been applied to vibration problems. This section present the extension of the theory to the case of a structural-acoustic problem.

From this point, the structural-acoustic system, as described in the previous section will be named mean structural-acoustic system in opposition to the stochastic structural-acoustic system described below.

\section{FACTORIZATION OF THE REDUCED MATRICES OF THE MEAN STRUCTURAL-ACOUSTIC SYSTEM}

In order to take into account random uncertainties with the proposed method, one has to factorize the generalized matrices of the mean model. In a second step, random matrices will be constructed.

\section{Factorization of the structural or cavity reduced matrices} of the mean model

In this section, we consider structural stiffness uncertainties. The case of structural mass, structural damping or acoustic cavity uncertainties will follow the same modelling process, that is valid for any positive definite matrix.

One can factorize the real, symmetric stiffness matrix by the Cholesky factorization

$$
\underline{\mathcal{K}}^{S}=\underline{L}_{\mathcal{K}^{S}}{ }^{T} \underline{L}_{\mathcal{K}^{S}}
$$

Since this stiffness matrix is usually diagonal, $\underline{L}_{\mathcal{K}^{s}}$ is also a diagonal matrix which terms are the square root of diagonal terms of $\underline{\mathcal{K}}^{S}$.
Factorization of the reduced vibroacoustic coupling $\underline{\text { matrix }}$

Let $\underline{C}$ be a the rectangular vibroacoustic coupling matrix. In any case, it can be written as:

$$
\underline{C}=\underline{U} \underline{T}
$$

where $\underline{U}$ has the same dimension as $\underline{C}$ and $\underline{U^{T}} \underline{U}=I$ (see Ref. [21]).

Let $\Sigma^{1 / 2}$ be the matrix of the singular values of $\underline{C}$, such that $\underline{C}^{T} \underline{C}=\Theta \Sigma \Theta^{T}$. Defining $\underline{T}$ as $\underline{T}=\Theta \Sigma^{1 / 2} \Theta^{T}$, we have $\underline{U}=\underline{C} \underline{T}^{-1}$. Finally, the factorization of $\underline{T}$, leads to the following factorization of $\underline{C}$ :

$$
\underline{C}=\underline{U} \underline{T}=\underline{U} \underline{L}_{C}^{T} \underline{L}_{C} \text { where } \underline{L}_{C}=\Sigma^{1 / 4} \Theta^{T}
$$

\section{Construction of the random matrices}

The nonparametric probabilistic approach of uncertainties consists in substituting the generalized matrices of the mean model by random matrices which construction laws are perfectly defined by the theory (see Refs. [19, 20, 21]). The reduced random structural stiffness matrix is constructed as:

$$
\mathbf{K}^{S}=\underline{L}_{\mathcal{K}^{S}}^{T} \mathbf{G}_{\mathcal{K}^{S}} \underline{L}_{\mathcal{K}^{S}}
$$

The same procedure leads to the reduced random coupling matrix :

$$
\mathbf{C}=\underline{U} \underline{L_{C}^{T}} \mathbf{G}_{C} \underline{L}_{C}
$$

if $\mathbf{G}$ denotes $\mathbf{G}_{\mathcal{K}^{s}}$ or $\mathbf{G}_{C}$ then $\mathbf{G}$ is a random matrix which mathematical expectation is the identity matrix: $E\{\mathrm{G}\}=I$.

The variance of the random matrix $\mathbf{G}$, is controlled by the parameter $\delta$, defined by:

$$
\delta=E\left\{\sqrt{\frac{1}{n}\|\mathbf{G}-I\|_{F}^{2}}\right\}
$$

where $n$ is the dimension of $\mathbf{G}$ and where $\|\cdot\|_{F}$ is the Frobenius norm.

\section{STOCHASTIC PROCESS IMPLEMENTATION}

The basic available information in order to build a stochastic process, is constituted of the mean structural mass, the mean structural damping, the mean structural stiffness, the mean vibro-acoustic coupling, the mean 
fluid mass, the mean fluid damping and the mean fluid stiffness.

For instance,

$$
E\left\{\mathbf{K}^{S}\right\}=\underline{\mathcal{K}}^{S}, E\{\mathbf{C}\}=\underline{C}
$$

The following algebraic representation of a random matrix $\mathbf{G}$, allows an easy construction of its random realizations. By definition,

$$
\mathbf{G}=\mathbf{L}_{\mathbf{G}}^{T} \mathbf{L}_{\mathbf{G}}
$$

in which $\mathbf{L}_{\mathbf{G}}$ is a random upper triangular real matrix which random elements are independent random variables defined as follows:

1. for $j<j^{\prime}$, the real-valued random variable $\left[\mathbf{L}_{\mathbf{G}}\right]_{\mathrm{jj}}$ is written as $\left[\mathbf{L}_{\mathbf{G}} \mathbf{l}_{\mathbf{j} j}=\sigma_{n} U_{j j}\right.$, in which $\sigma_{n}=\delta(n+1)^{-1 / 2}$ and where $U_{i j}$, is a real-valued Gaussian random variable with zero mean and variance equal to $1 . \delta$ is the uncertainty parameter that controls the actual matrix variance,

2. for $j=j^{\prime}$, the positive-valued random variable $\left[\mathbf{L}_{\mathbf{G}}\right]_{\mathrm{jj}}$ is written as $\left[\mathbf{L}_{\mathbf{G}}\right]_{\mathbf{j} j^{\prime}}=\sigma_{n} \sqrt[2 V_{j}]{ }$ in which $V_{j}$ is a positive-valued gamma random variable whose probability density function $p_{V j}$ with respect to $d v$ is written as

$$
p_{V_{j}}(v)=\mathbf{1}_{R^{+}}(v) \frac{1}{\Gamma\left(\frac{n+1}{2 \delta^{2}}+\frac{1-j}{2}\right)} v\left(\frac{n+1}{2 \delta^{2}} \frac{1-j}{2}\right) e^{-v}
$$

Eq.(11) is then replaced by the following random linear system



where $\mathbf{A}^{S}, \mathbf{A}^{F}$, and $\mathbf{C}$ are random matrices constructed according to the process described above .

The random displacement field of the structure and the random pressure field inside the internal acoustic cavity are then computed by using the following equations, derived from Eqs.(9) and (10)):

$$
\begin{gathered}
\mathbf{U}^{S}(\omega)=[\Psi] Q^{S}(\omega) \\
\mathbf{P}^{F}(\omega)=i \omega[\underline{\Phi}] \widetilde{Q}^{F}(\omega)
\end{gathered}
$$

For each realization of the stochastic process, Eq.(12) is solved for every $\omega$.

\section{CONFIDENCE REGION OF A RANDOM FREQUENCY RESPONSE}

Managing results of a random process is an important issue when a large number of results has to be condensed into an understandable data set. Individual results will never be presented since they do not have any special interest: only statistics of the stochastic process result are relevant. Since vibroacoustic responses include resonances and anti-resonances random uncertainties may produce different kind of data distributions. Therefore, the use of confidence intervals is preferred in order to describe any kind of results distribution.

Let $V(\omega)$ be the modulus of any observed frequency response -either the modulus of a displacement frequency response on the structural part or the modulus of an acoustic frequency response in the cavity-. The confidence region associated with the probability level $P_{C}$ for the random function $\{V(\omega), \omega \in B\}$ is constructed using quantiles. For fixed $\omega$ in $B$, let $F_{V(\omega)}$ be the cumulative distribution function (continuous from the right) of random variable $V(\omega)$ which is such that $F_{V(\omega)}(w)=P(V(\omega)<w)$ where $P$ means probability.

For $0<q<1$, the q-th quantile or fractile of $F_{V(\omega)}$ is defined as

$$
\xi(q)=\inf \left\{w: F_{V}(\omega)(w) \geq q\right\}
$$

The upper envelope $v^{+}(\omega)$ and the lower envelope $v^{-}(\omega)$ of the confidence region the probability level $P_{C}$ are defined by:

$$
v^{+}(\omega)=\xi\left(P_{C}\right), \quad v^{-}(\omega)=\xi\left(1-P_{C}\right)
$$

The estimation of $v^{+}(\omega)$ and $v^{-}(\omega)$ is performed by using the sample quantiles [22].

Let $v_{1}(\omega)=V\left(\omega ; \theta_{1}\right), \ldots \ldots . ., v_{n_{r}}(\omega)=V\left(\omega ; \theta_{n_{r}}\right)$ be the $n_{r}$ independent realizations of the random variable $V(\omega)$. Let $\widetilde{v}_{1}(\omega)<\ldots .<\widetilde{v}_{n_{r}}(\omega)$ be the ordered statistics associated with $v_{1}(\omega), \ldots, v_{n_{r}}(\omega)$. The estimations of the upper and lower envelopes of the confidence region are then computed as:

$$
\begin{array}{ll}
v^{+}(\omega)=\widetilde{v}_{j^{+}}(\omega), & j^{+}=\operatorname{fix}\left(n_{r} \cdot P_{C}\right) \\
v^{-}(\omega)=\widetilde{v}_{j^{-}}(\omega), & j^{-}=\operatorname{fix}\left(n_{r} \cdot\left(1-P_{C}\right)\right)
\end{array}
$$

in which $\operatorname{fix}(x)$ is the integer part of the real number $x$.

\section{APPLICATION TO THE VIBROACOUSTIC RESPONSE OF A TRIMMED BODY}

\section{MEAN MODEL OF THE TRIMMED BODY}

The mean model considered is a Finite Element Model of a trimmed body as currently built by PSA PeugeotCitroën engineers (see Figure2). The model consists in a detailed Finite Element model of the Body In White, trimmed with either detailed or simplified models of trim components (see Ref. [23]). This model is used to study 
the frequency response functions in the low-frequency range $-\mathrm{B}=[34,200] \mathrm{Hz}-$, for a given set of external loads induced by the powertrain (12 loaded structural DOF). This load case simulates the main contributions to the booming noise.

The finite element model of the structure has 712000 DOF. The internal acoustic cavity contains the seats. The finite element model of the internal acoustic cavity has 2686 DOF. It should be noted that the two meshes are highly incompatible on the fluid-structure coupling interface.

For practical reasons -such as availability of data-, part of the trimmed body, the cavity geometry or the fluid/structure interface are simplified. The degree of simplification may vary, depending on the time of the design process when the model is used. These simplifications individually introduce errors, that, when combined, seem to behave randomly. Effect of simplifications can then only be predicted through a stochastic modelling.

The purpose of this section is to show, using the nonparametric modelling, how the trimmed body model is sensitive to random uncertainties associated to the structural part, to the cavity, and to the coupling interface. But first the convergence of the numerical stochastic process has to be studied.



Figure 2 Finite element model of the trimmed body

\section{CONVERGENCE STUDY OF THE MONTE-CARLO SIMULATION}

The random Eq.(12) is solved by a Monte Carlo numerical simulation. The convergence of the simulation is studied here in the applicative context. Two parameters have been investigated: the number of realizations $n_{r}$, and the number of modes considered in both structural part and cavity. Provided modal series convergence is governed by eigenfrequencies, the number of modes in the series will be indicated by the highest eigenfrequency considered. We indicate that correction methods such as the residual vectors are not suitable in the present case, and convergence is only due to the natural decrease of high order modes contributions.

The convergence of the random solution is analyzed in studying the graph of the function $\operatorname{conv}^{H}\left(n_{r}\right)$ defined by:

$$
\operatorname{conv}^{H}\left(n_{r}\right)=\frac{1}{n_{r}} \sum_{l=1}^{n_{r}} \int_{B}\left\|\mathbf{Q}^{H}\left(\omega ; \theta_{l}\right)\right\|^{2} d \omega
$$

in which exponent $H$ is either $S$ for the structure or $F$ for the fluid and where $\mathbf{Q}^{H}\left(\omega ; \theta_{1}\right) \ldots . \mathbf{Q}^{H}\left(\omega ; \theta_{n_{r}}\right)$ are the $n_{r}$ realizations of the vector-valued random variable $\mathbf{Q}^{H}(\omega)$, solution of Eq.(12)

This convergence indicator is in fact the mean energy of either the structural part or the internal acoustic cavity, in the frequency band $B$.

Various combinations of modal truncation for the structural part and cavity have been considered. Figure3 displays the graph of the function $\operatorname{conv}^{S}\left(n_{r}\right)$ for the structure. Figure 4 displays the graph of the function $\operatorname{conv}^{F}\left(n_{r}\right)$ for the internal acoustic cavity.

From these two figures, it can be seen that a reasonable mean-square convergence of the random structural-acoustic system is reached for :

- $n_{r}=600$ realizations

- $n$ structural modes corresponding to a modal truncation at $400 \mathrm{~Hz}$

- $m$ acoustic modes corresponding to a modal truncation at $350 \mathrm{~Hz}$

These values will be used in what follows.

It may be noticed that the convergence is obtained for a modal truncation frequency that is rather high. This is due to the fact that the modal basis of the mean model is used as a functional -non modal- basis to build the response of the random systems. High order terms are then required to fit the local modifications of the mean modal shapes.

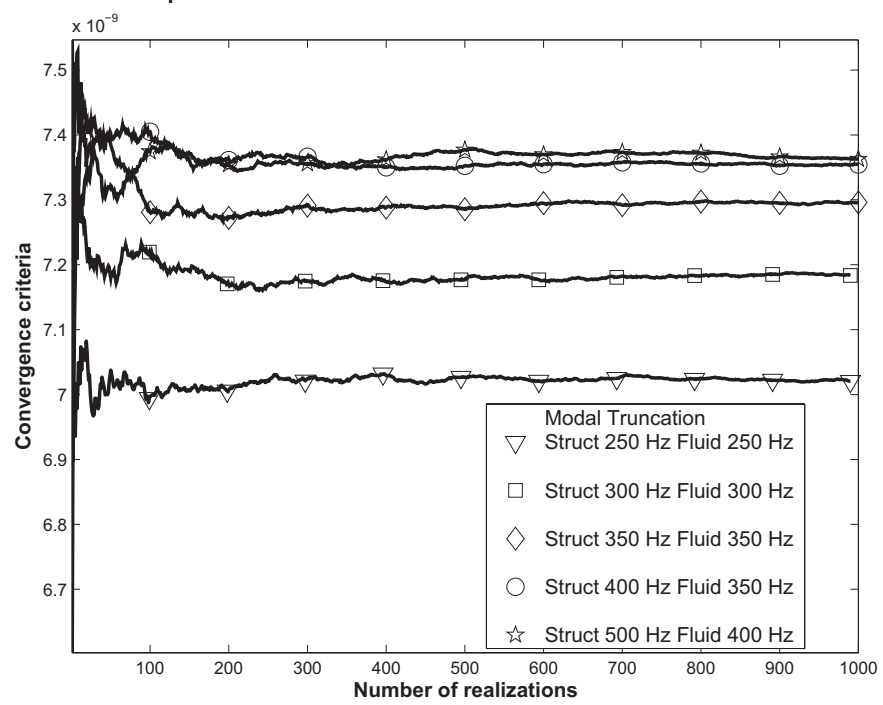

Figure 3 Graph of the function $n_{r} \rightarrow$ conv $S_{(n r)}$ related to the convergence of the random structural generalized coordinates. 


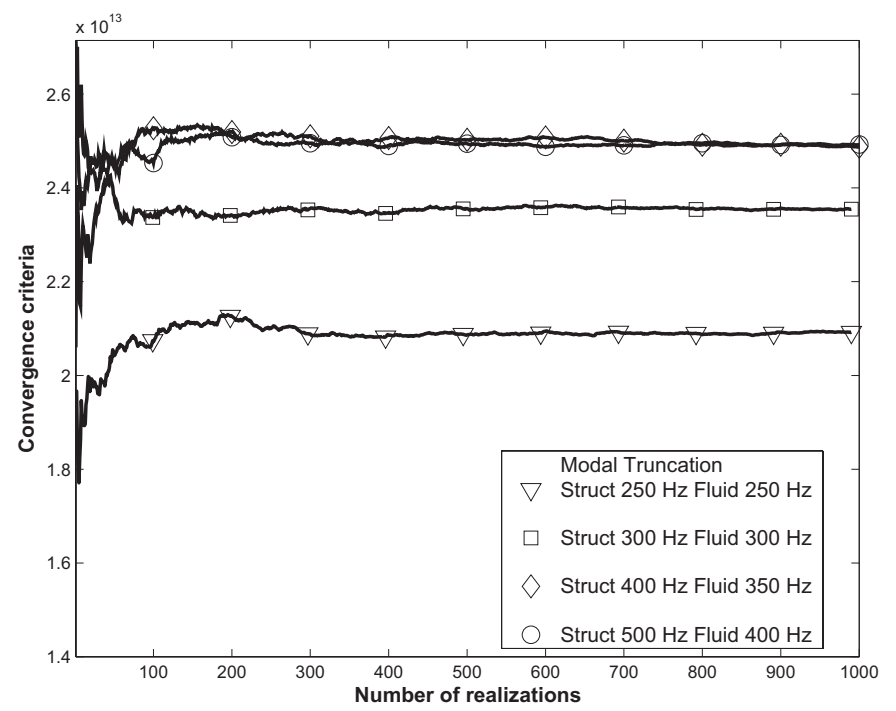

Figure 4 Graph of the function $n_{r} \rightarrow \operatorname{conv} v^{F}(n r)$ related to the convergence of the random acoustic generalized coordinates.

\section{RANDOM VIBROACOUSTIC FREQUENCY RESPONSES OF THE TRIMMED BODY MODEL SUBJECT TO RANDOM UNCERTAINTIES}

In this section, we will examine separately the sensitivity of the dynamic responses to the individual causes of uncertainties we have modelled. The uncertainty parameters has been set to a value such as the order of magnitude of the results spread seems realistic.

Results are all presented in the same way. The thick solid line is the graph of the response of the mean model (original model), the thick dashed line is the mean value of the random frequency response and the grey region is the confidence region for a probability level $P_{C}=95 \%$.

\section{Effects of random structural uncertainties on frequency responses}

As any real process, the manufacturing process causes deviations regarding the designed product. These deviations may be random like for example the structural properties of foams, but they are often systematic such as thickness and curvature modifications imposed by the stamping process. In this case, the proposed method will be relevant if it is assumed that these modifications have no coherent effect on the structure behaviour. Another cause of uncertainty when modeling is the diversity that comes from the fact that vehicles are declined in several equipment versions. Moreover, due to simultaneous engineering, the exact definition of these equipments is not available when it is required for computation.

Last but not least, the lack of modelling knowledge remains a major cause of uncertainties.

In this sub-section, the coupling operator and internal acoustic cavity operator remain deterministic. Only the stiffness matrix of the structural part is a random matrix and the uncertainty parameter is the same for all presented results.

Figure 5 displays the structural frequency response in the $z$-direction at point 1 located on the vehicle framework (see Fig.2). It can be seen a narrow confidence region (a few decibels) with a tendency to a slight increase with frequency.

Figure 6 displays the structural frequency response in the normal direction at point 2 located on the car roof. This is a flexible structural element. It can be seen that the confidence region is much larger than in the previous case, up to $20 \mathrm{~dB}$. The width of the confidence region seem to increase progressively and stabilize above 120 $\mathrm{Hz}$.

Figure 7 displays the structural frequency response in the normal direction at point 1 located on the firewall. The width of the confidence region is about $5 \mathrm{~dB}$ at lower frequency and increase suddenly above $140 \mathrm{~Hz}$, as if the behaviour was switching form the one of figure 5 (stiff) to the one of figure 6 (flexible).

From these 3 figures, it can be concluded that the same random uncertainty model -controlled by a single number- produce a variety of effects depending on the location of observation points, and frequency range.

Figure 8 shows the effect of the structural uncertainty on the acoustic pressure at the driver's ear position in the cavity. The width of the confidence region increases regularly with frequency but remains lower than what was observed on the roof.



Figure 5 Confidence region of the random frequency response for the structural $z$ displacement (point 1) structural uncertainties. Mean model prediction (thick solid line), mean response of the stochastic model (dashed line), confidence region (grey region). 


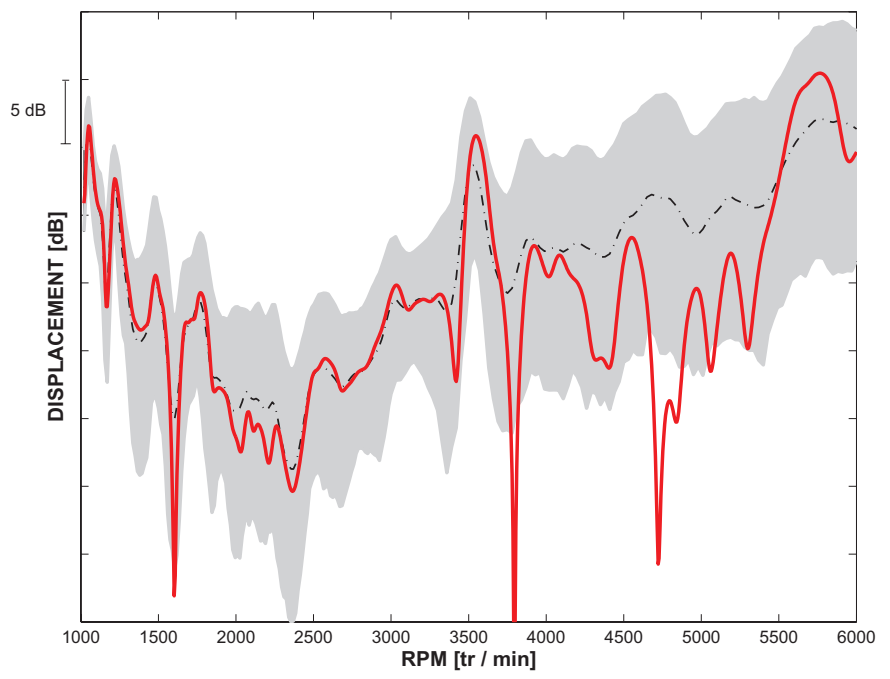

Figure 6 Confidence region of the random frequency response for the structural normal displacement (point 2) structural uncertainties. Mean model prediction (thick solid line), mean response of the stochastic model (dashed line), confidence region (grey region).

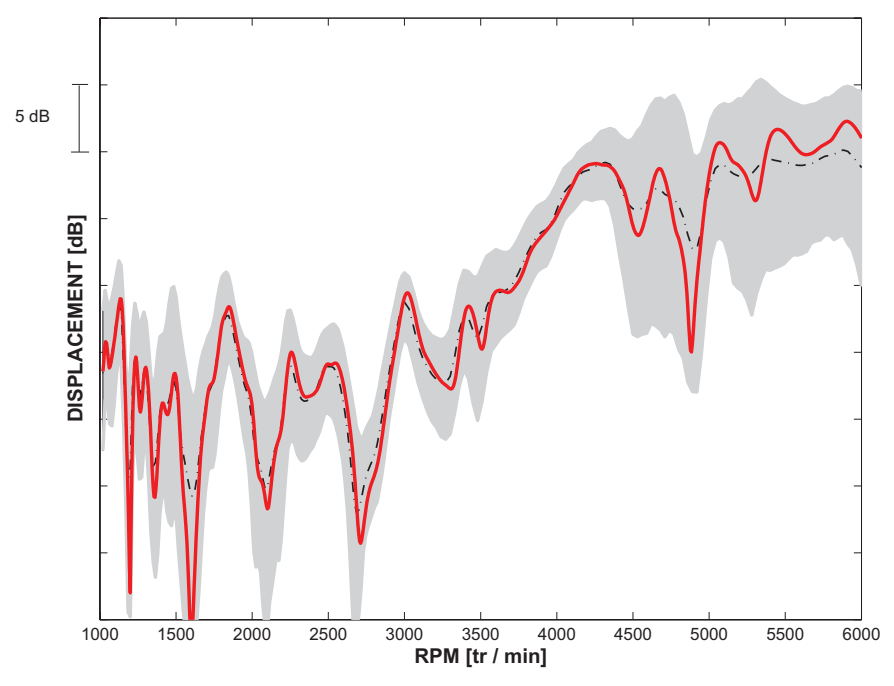

Figure 7 Confidence region of the random frequency response for the structural normal displacement (point 3) structural uncertainties. Mean model prediction (thick solid line), mean response of the stochastic model (dashed line), confidence region (grey region).

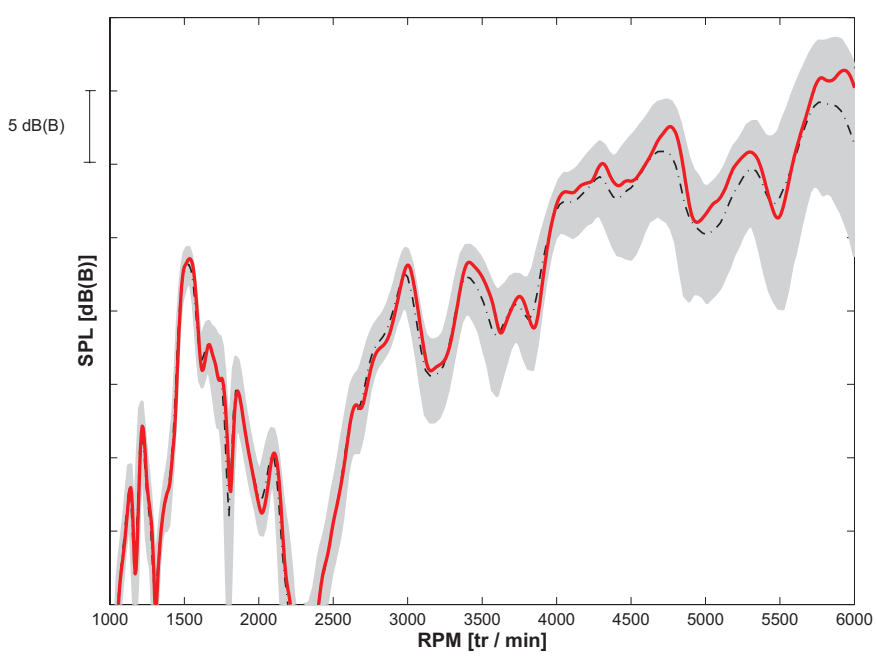

Figure 8 Confidence region of the random frequency response for the internal acoustic pressure at a point located at the driver's ear for structural uncertainties. Mean model prediction (thick solid line), mean response of the stochastic model (dashed line), confidence region (grey region).

\section{Effect of random structural uncertainties on modal shapes}

The previous results are the consequence of the modal basis changes induced by the uncertainty modelling. Therefore, it seemed interesting to examine statistically the modal shapes and eigenfrequencies of the trimmed body vibration modes.

We first introduce the Stochastic Modal Assurance Criteria (SMAC) between the mean model structural modes and structural random modes, defined as:

$$
\left[\operatorname{SMAC}\left(\theta_{l}\right)\right]_{i j}=\frac{\left\langle\underline{\Psi}_{i}, \mathbf{Y}_{j}\right\rangle^{2}}{\left\langle\underline{\Psi}_{i}, \underline{\Psi}_{i}\right\rangle\left\langle\mathbf{Y}_{j}, \mathbf{Y}_{j}\right\rangle}
$$

where $[\Psi]$ are the mean model structural mode shapes and $[\mathbf{Y}]$ are the structural random mode shapes.

The SMAC function is a random variable, which mathematical expectation is estimated as :

$$
E\left\{[\mathbf{S M A C}]_{i j}\right\}=\frac{1}{n_{r}} \sum_{l=1}^{n_{r}}\left[\mathbf{S M A C}\left(\theta_{l}\right)\right]_{i j}
$$

Figure 9 displays an estimation of the Stochastic Modal Assurance Criteria mathematical expectation for a given level of structural uncertainty. 

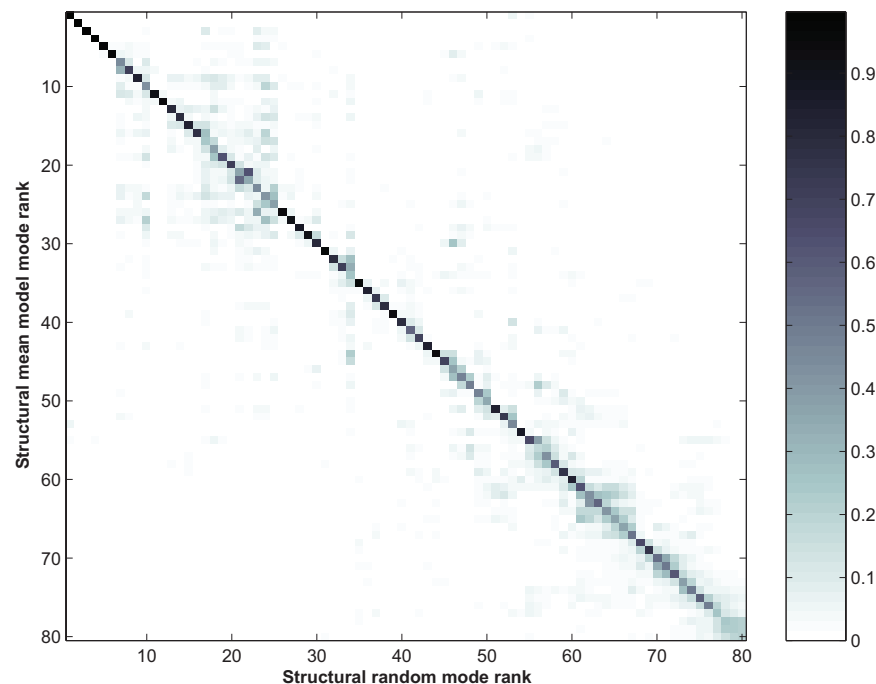

Figure 9 (a) Estimation of the mathematical expectation of SMAC from rank mode 1 to rank mode 80 .

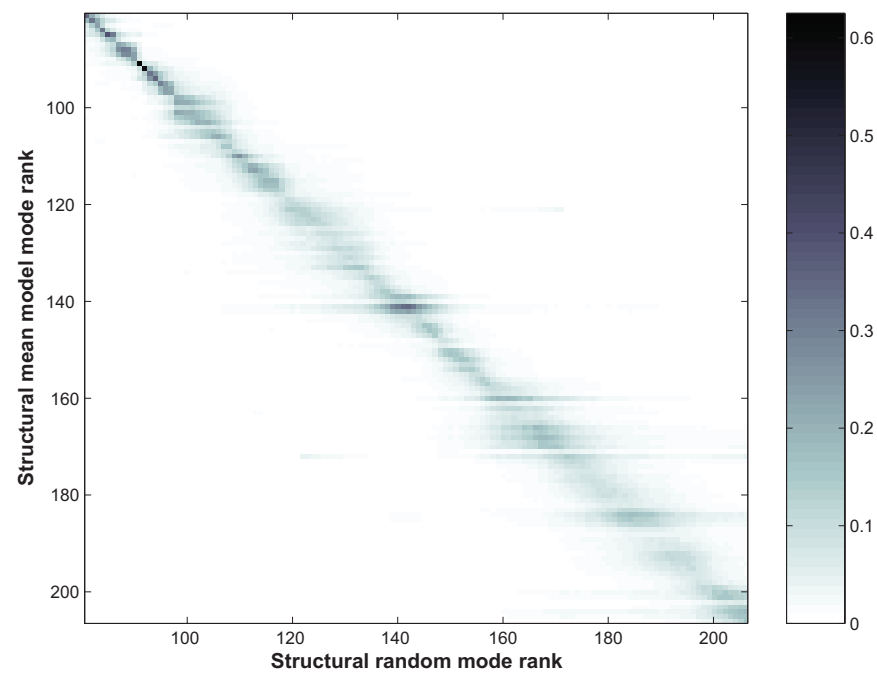

Figure 9 (b) Estimation of the mathematical expectation of SMAC from rank mode 81 to rank mode 200 .

In the first part of the frequency range, up to structural mode rank 80 , one can observe a low sensitivity of modal shapes: SMAC matrix is close to identity matrix meaning random modal shapes are close to the mean model modal shapes. In this case, it is meaningful to plot the eigenfrequencies distribution. As an example, Figure 10 displays an estimation of the probability density function of the first body torsion mode relatively to the mean model torsion eigenfrequency.

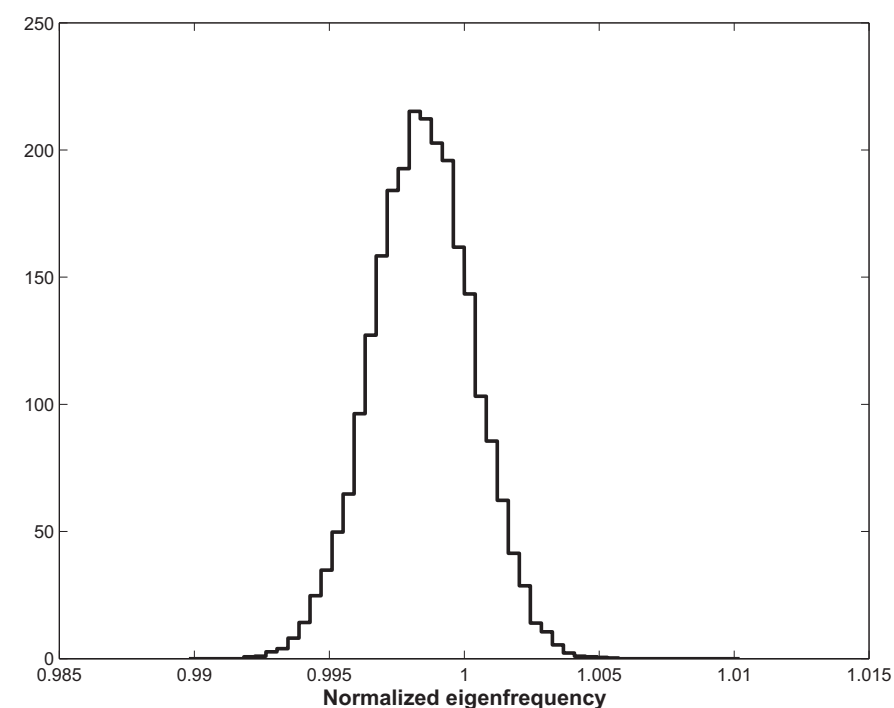

Figure 10 Estimation of the probability density function of the fisrt torsional body mode with respect to its eigenfrequency.

Above structural mode rank 80 , the SMAC matrix is not diagonal anymore, indicating that structural modes shapes are highly sensitive to structural random uncertainties. It is then impossible to track modes and individual eigenfrequency statistics become meaningless. This is a typical feature of the medium/high frequencies vibroacoustic models, such as SEA, that only consider modal densities and assume no peculiarity of individual modes.

It is recalled here that the limit between the two behaviours is depending on the uncertainty parameter that was nearly arbitrary here. Nevertheless, these results present globally the same trends as observed experimentally: low order modes appear with stable shapes while high order modes may be difficult to capture.

\section{Effect of Vibroacoustic coupling interface uncertainties}

In this section, the case of the coupling interface random uncertainties will be discussed. These uncertainties are mainly related to modelling uncertainties due to the incompatibility between the structural mesh and the cavity mesh. They may also account for geometrical uncertainties of both meshes.

Structural responses appear to be nearly insensitive to the coupling uncertainties.

Figure 11 displays the acoustic frequency response for the internal acoustic pressure at the driver's ear position, when considering only coupling interface uncertainty. Again in this case, the confidence region is first increasing with frequency, and then -approximately above $60 \mathrm{~Hz}$ - becomes nearly independent of the frequency. 


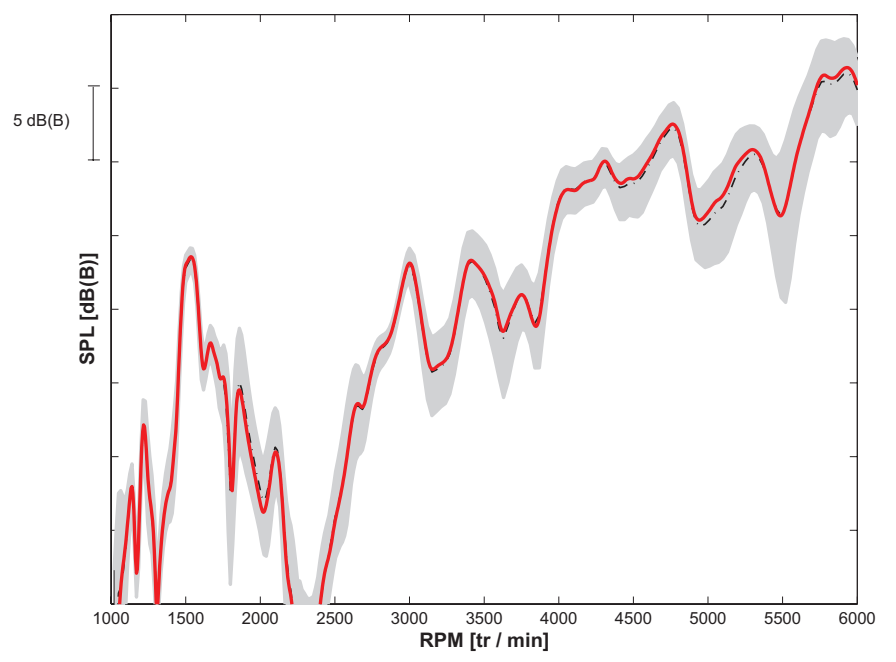

Figure 11 Confidence region of the random frequency response for the internal acoustic pressure at a point located at the driver's ear for vibroacoustic coupling uncertainties. Mean model prediction (thick solid line), mean response of the stochastic model (dashed line), confidence region (grey region).

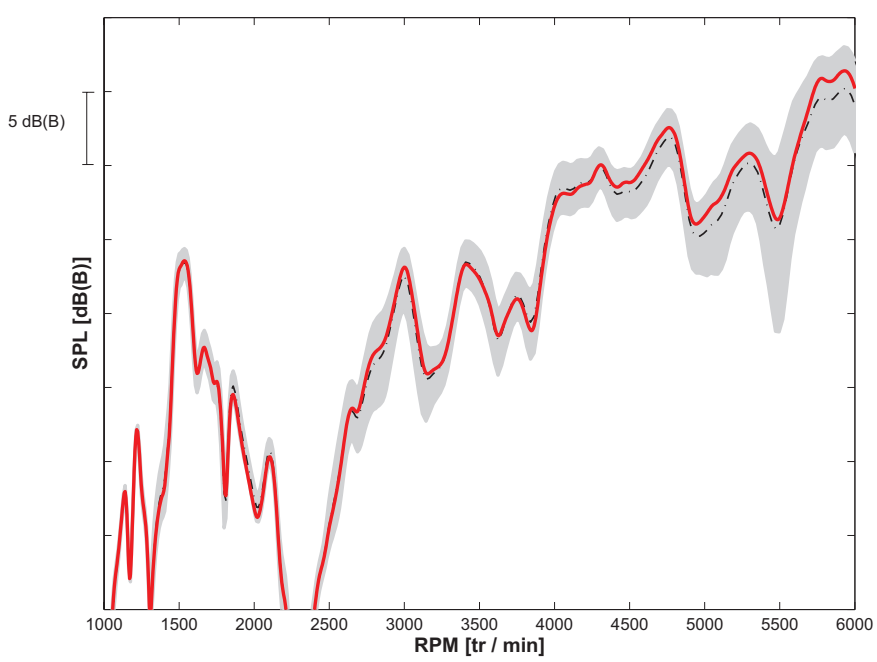

Figure 13 Confidence region of the random frequency response for the internal acoustic pressure at a point located at the driver's ear for acoustic cavity uncertainties. Mean model prediction (thick solid line), mean response of the stochastic model (dashed line), confidence region (grey region).

\section{CONCLUSION}

A new approach to investigate the sensitivity of low frequencies vibroacoustic responses to random uncertainties has been presented and applied to a current trimmed body model. The proposed method, based on the maximum of entropy principle, models randomly the variability of a vibroacoustic system. The range of the variability is controlled by a reduced number of parameters associated to the structural part, the cavity, and the coupling interface. Any cause of random uncertainty may be described using this method: data uncertainties as well as modelling uncertainties. Assuming modelling errors is the major cause of the lack of predictability of vehicle models, the proposed method is able to provide, at least, the sensitivity of vibroacoustic responses to such errors, until errors are quantified which will permit a robust prediction.

The numerical implementation is performed using random matrices and a Monte-Carlo simulation. Random matrices are constructed using the available information i.e. the FE model of the studied problem, named mean model. The method is numerically efficient, when generalized coordinates are used for the cavity and the structural part. Results shown are confidence regions of the Frequency Response Functions obtained with a probability of $95 \%$. It should be noted that all the realizations of the Monte-Carlo simulation remain physically consistent. This important feature is characteristic of the theory (see Ref. [21]).

The application to a trimmed body have demonstrated the possibility to implement the method within the existing vibro-acoustic modelling process at PSA Peugeot-Citroën. Results of computations show the 
sensitivity of the booming noise to structural and to cavity uncertainties as well as coupling interface uncertainties. It is observed that stiff parts of the vehicle (framework) are more robust to structural random uncertainties than flexible part (panels). This seems to fit quiet well what may be observed on car bodies: global structural modes are usually less dispersed than local modes. Another illustration of the random uncertainty modelling is the mean value Stochastic MAC table between the mean model and the random model. This table shows that modal shapes can not be recognized any more above a given frequency. Again this result is close to observations that led engineers to use statistical modal methods, such as SEA, to study the so-called high frequency range.

In order to go further in the practical use of the presented theory, and for example provide robust predictions, one has to identify the uncertainty parameters of the nonparametric probabilistic approach. Two different experimental approaches may be considered:

1. when dispersion measurements are available, one can identify the uncertainty parameters that provide the computed spread the closer to the measured one. This procedure is efficient to identify the amount of uncertainty related to the vehicle diversity, manufacturing dispersion, environmental and operational causes, but it requires a good quality mean model.

2. when only one experiment is available, or considering the mean value of a statistical observations, one can increase the uncertainty parameters until the predicted envelope covers the measured result. The obtained value is a measure of the distance between the mean model and the targeted experiment. This procedure is clearly dedicated to the identification of modelling errors.

Work is in progress in these ways.

\section{REFERENCES}

[1] Hills E., Mace B.R., Ferguson N.S. (2004), Response statistics of stochastic built-up structures ISMA proceedings Cdrom, Leuven, Belgium

[2] Kompella M.S., Bernhard R.J. (1996), Variation of structural-acoustic characteristics of automotive vehicles

Noise Control Engineering Journal pp93-99

[3] Moeller, M. J., Thomas, R. S., Chen, S.CAE, NAFEMS QAWG, Quality System Supplement to ISO 9001 Relating to Finite Element Analysis in The Design and Validation of Engineering Products, Issue 1.3, 2 July 1993.

[4] Chandra, N. and Lenk, P., NVH CAE Quality Metrics, SAE Noise and Vibration Conference, SAE Paper 199901-1791, Traverse City, Michigan, May 1999.
[5] Ghanem R., Spanos P.D. (1991), Stochastic Finite Elements: A spectral Approach, Springer-Verlag,New York.

[6] Germain, P (1973). Cours de Mécanique des Milieux Continus\}. Mason, Paris.

[7] Marsden, J.E, and Hugues, T. J. R. (1983).

Mathematical Foundations of Elasticity. Prentice Hall, Englewood Cliffs, New Jersey.

[8] Ciarlet, P.G (1988). Mathematical Elasticity, Vol.I: Three-dimensional Elasticity . North Holland,

Amsterdam.

[9] Ohayon, R., Soize, C. Structural Acoustics And

Vibration\}, Academic Press

[10] Landau, L. and Lifchitz, E. (1992b). Fluid

Mechanics. Pergamon Press, Oxford

[11] Lighthill, J. (1978). Waves in Fluids. Cambridge University Press, MA.

[12] Pierce, A. D. (1989). Acoustics: An Introduction to its Physical Principles and Applications. Acoust. Soc Am. Publications on Acoustics, Woodbury, NY, U.S.A. (originally published in 1981, McGraw-Hill, New York). [13] Zienkiewicz, 0. C. and Taylor, R. L. (1989). The Finite Element Method. McGraw-Hill, New York

, 4th edn. (vol. 1, 1989 and vol. 2, 1991

[14] Lesueur, C. (1989). Rayonnement Acoustique des Structures. Eyrolles, Paris.

[15] Dautray, R. and Lions, J. L. (1992). Mathematical Analysis And Numerical Methods for Science and Technology. Springer-Verlag, Berlin

[16] Meirovitch, L. (1990). Dynamics and Control of Structures. Wiley, New York.

[17] Argyris, J. and Mlejnek, H.P. (1991). Dynamics of Structures. North-Holland, Amsterdam.

[18] Bathe, K. J. and Wilson , E.L. (1976). Numerical Methods in Finite Element Analysis. Prentice-Hall, New York.

[19] Soize C. (2000), A Non-parametric Model of Random Uncertainties on Reduced Matrix Model in Structural Dynamics, Probabilistic Engineering Mechanics, Vol. 15, No. 3, pp. 277-294

[20] Soize C. (2001), Maximum entropy approach for modeling random uncertainties in transient elastodynamics, J. Acoust. Soc. Amer., Vol. 109, No. 5, pp. 1979-1996

[21] Soize C. (2005), Random matrix theory for modeling uncertainties in computational mechanics, Computer Methods in Applied Mechanics and Engineering, Vol. 194, pp. 1333-1366

[22] Serfling R.J. (1980), Approximation Theorems of Mathematical Statistics, John Wiley $1 \&$ Sons [23] Sol A., Van Herpe F. (2001), Numerical prediction of a whole car vibro-acoustics behaviour at low frequencies, SAE Noise and Vibration Conference, SAE Paper 2001-01-1521, Traverse City, Michigan, May 2001

\section{CONTACT}

laurent.gagliardini@mpsa.com 